\title{
Stratégies paysannes de participation à la domestication du poivre sauvage de Madagascar, le Tsiperifery
}

\author{
Sedera Norotiana Rasambo ${ }^{1, *}$, Jérôme Queste ${ }^{2,3}$, Jules Razafiarijaona ${ }^{4}$, Sarah Audouin ${ }^{3,5}$, \\ Frédérique Jankowski ${ }^{2,3}$, Tahiry Rabefarihy ${ }^{4}$ et Romaine Ramananarivo ${ }^{4}$ \\ ${ }^{1}$ École Doctorale Gestion des Ressources Naturelles et Développement, École Supérieure des Sciences Agronomiques, Université \\ d'Antananarivo, Antananarivo, Madagascar \\ 2 CIRAD, UMR SENS, F-34398 Montpellier, France \\ ${ }^{3}$ Univ Montpellier, CIRAD, Montpellier, France \\ ${ }^{4}$ École Supérieure des Sciences Agronomiques, Université d'Antananarivo, Antananarivo, Madagascar \\ ${ }^{5}$ CIRAD, UMR Innovation, F-34398 Montpellier, France
}

\begin{abstract}
Résumé - À Madagascar, le Tsiperifery, une espèce de poivrier sauvage endémique de l'île, est actuellement soumis à une surexploitation. Un programme participatif de domestication de cette plante a été lancé pour assurer la durabilité de son exploitation. Des techniques de culture sont co-construites par des chercheurs et des paysans sur trois sites pilotes. Cette étude s'intéresse aux facteurs influençant la propension des paysans à co-construire et expérimenter ces techniques. Les données collectées via 90 entretiens semi-dirigés ont été traitées en suivant les principes de l'approche "grounded theory». Les principaux déterminants influençant le comportement des paysans sont (i) la logique économique, (ii) le contexte informationnel, (iii) l'accès au marché, (iv) les capacités productives de l'exploitation et (v) l'esprit de conservation de la nature. À partir de ces déterminants, nous avons pu définir cinq types de stratégies paysannes : les « homo-economicus »; les « conservationnistes »; les « opportunistes », les « exclus » et les « suiveurs ». Cette meilleure compréhension des stratégies paysannes permet d'améliorer la conception et la conduite des programmes de recherche participative.
\end{abstract}

Mots clés : innovation / recherche participative / produits forestiers non ligneux / technique de culture / stratégies paysannes

\begin{abstract}
Farmers' strategies in a participatory research program on the Madagascar wild pepper (Tsiperifery) domestication. In Madagascar, Tsiperifery, a wild pepper species endemic to the island, is currently undergoing overexploitation. A participatory domestication program is operating to ensure its sustainable exploitation. Cultivation techniques are co-constructed by researchers and farmers in three pilot sites. This study focuses on the factors influencing the behavior of farmers to experiment and co-construct these techniques. Data from 90 semi-structured interviews was analyzed using a "grounded theory" approach. Results show that factors influencing behaviors are (i) the economic rationale, (ii) the information environment, (iii) the access to the market, (iv) the farm productive capacity and (v) the conservation spirit. Using these determinants, farmers' strategies could be classified in five types: "homo-economicus", "conservationists", "opportunists", "outsiders" and "followers". A better understanding of farmers' strategies may improve the design and operation of participatory research programs.
\end{abstract}

Keywords: innovation / participatory research / non-timber forest products / cultivation technique / farmers' strategies

\section{Introduction}

Depuis plusieurs décennies, de nombreux acteurs de la conservation et du développement promeuvent l'exploitation

\footnotetext{
*Auteur de correspondance : sederanorotiana@gmail.com
}

des produits forestiers non ligneux - PFNL (Vantomme et Gazza, 2010). Ces actions sont fondées sur l'idée selon laquelle donner une valeur économique aux PFNL constitue une incitation à la conservation des écosystèmes naturels (Kusters et al., 2006). Cependant, l'exploitation des PFNL ne permet de concilier conservation et développement local que 
sous certaines conditions (Arnold et Ruiz Pérez, 2001 ; Belcher et al., 2005). Plusieurs études de cas montrent que l'exploitation non contrôlée de ces produits a non seulement menacé d'extinction les espèces concernées mais a aussi fortement dégradé leurs habitats (Kusters et al., 2006). L'essentiel des cas étudiés portent sur des PFNL sauvages, cueillis dans des forêts communes (Tchatat et Ndoye, 2006).

Des solutions organisationnelles comme des quotas de cueillette ou la restriction des dates de campagne peuvent réguler ces pressions (Belcher et al., 2005). Mais ces solutions se révèlent difficiles à mettre en œuvre. Leur acceptabilité sociale reste faible et elles nécessitent des moyens importants, dont les pays du Sud sont généralement dépourvus (Bertrand et al., 2014). À Madagascar, les expériences passées de l'exploitation de l'écorce du Prunus africana (Stewart, 2003) et du caoutchouc (Danthu et al., 2016) illustrent ces difficultés. Des alternatives, fondées sur la cueillette aménagée et la culture semi-intensive, peuvent se révéler plus vertueuses d'un point de vue environnemental, social et économique (Kusters et al., 2006).

Le Tsiperifery, un poivrier sauvage endémique de Madagascar, produit des baies suscitant l'intérêt de nombreux consommateurs sur le marché international (Razafimandimby et al., 2017); mais il fait l'objet de pratiques de cueillette non durables. Dans l'objectif d'assurer la durabilité de son exploitation, un collectif de chercheurs auquel appartiennent plusieurs co-auteurs de cet article, a initié en 2016 un programme de recherche participative visant à aboutir à la domestication de cette espèce. Ce programme est construit autour du concept «follow the technology» de Douthwaite et al. (2003) qui sera explicité plus loin dans cet article. La démarche adoptée place les paysans au cœur du processus d'innovation comme co-constructeurs et expérimentateurs de techniques en cours d'élaboration. Plusieurs questions se posent alors: Comment les paysans réagissent à cette proposition? Acceptent-ils d'expérimenter puis de co-construire les techniques de culture du Tsiperifery proposées par les porteurs du projet? Quels sont les déterminants de leur comportement et quels sont les facteurs pouvant bloquer leur participation au programme de domestication du Tsiperifery? Cet article s'intéresse aux stratégies des paysans concernant leur adhésion au processus d'innovation en cours de coconstruction et aux facteurs qui influencent ces comportements. Les résultats de cette analyse contribueront à alimenter les réflexions in itinere sur la conduite du programme de domestication du Tsiperifery, notamment le lien entre la production de connaissances techniques et la participation des paysans à différentes étapes du processus d'innovation.

\section{Matériels et méthodes}

\subsection{Matériels}

\subsubsection{Tsiperifery, le poivrier sauvage de Madagascar}

Le Tsiperifery, appelé communément «poivrier sauvage de Madagascar » appartient au genre Piper de la famille des Piperaceae. Il a pour habitat les forêts denses humides sempervirentes de l'est de Madagascar. Au moment où paraît cet article (avril 2021), la dernière révision taxonomique en date l'identifie comme Piper borbonense (Miq.) C.DC., 1869, mais il pourrait s'agir d'une nouvelle espèce, le Piper malgassicum (Palchetti et al., 2018). La plante est dioïque.

En termes de phénologie, aucune étude précise n'a encore été effectuée sur le Tsiperifery. Les informations apportées par des herbiers et les enquêtes auprès des cueilleurs et riverains des forêts indiquent que la floraison est annuelle et que la maturité des fruits est optimale d'octobre à décembre (Touati, 2012).

La figure 1 présente la liane de Tsiperifery sur tuteur et ses fruits, les baies de poivre.

Ce PFNL n'est pas encore soumis à une réglementation spécifique et est actuellement exporté en tant que poivre noir. Environ 50 t ont été exportées en 2011 et 25 t en 2014, à un prix de 100 à $200 € / \mathrm{kg}$ (Razafimandimby et al., 2017). La demande élevée a engendré une surexploitation de l'espèce et expose son habitat forestier à des risques de dégradation.

Les jeunes lianes de Tsiperifery poussent à l'ombre, mais une fois adultes elles ont besoin de lumière pour fleurir et fructifier. Les lianes adultes fructifient à plus de $10 \mathrm{~m}$ de hauteur. Pour atteindre les grappes de Tsiperifery, les cueilleurs coupent les tuteurs ou arrachent la liane (Razafimandimby et al., 2017). En conséquence, les pratiques de cueillette portent atteinte à la population des lianes, à la forêt qui les héberge et aux autres espèces endémiques qui s'y trouvent.

\subsubsection{Programme de domestication du Tsiperifery}

Le programme de domestication du Tsiperifery a été mis en œuvre à Madagascar à partir de 2016 par le dispositif de recherche en partenariat «Forêt et biodiversité à Madagascar» constitué par le Cirad, le Fofifa et l'Université d'Antananarivo. Le programme est mis en œuvre par l'intermédiaire de deux projets similaires, Capetsip et Dometsip (https://www.foretsbiodiv.org/projets/en-cours), sur trois sites pilotes. À cette date (avril 2021), toutes les connaissances nécessaires pour une domestication effective du Tsiperifery ne sont pas encore stabilisées: différents paramètres tels que la diversité génétique, les paramètres de croissance, la fertilisation, la fructification ou la protection contre les maladies sont mal maîtrisés. Face à la dégradation des forêts et compte tenu des faibles connaissances scientifiques sur cette espèce, les chercheurs ont adopté une démarche participative associant chercheurs et parties prenantes à la co-construction des techniques de culture de la liane, en s'appuyant sur le concept «follow the technology» proposé par Douthwaite et al. (2003). Cette approche constructiviste consiste à (i) concevoir, sur la base des connaissances scientifiques existantes, une première proposition d'itinéraire technique appelée "promesse plausible». Celle-ci est alors (ii) présentée aux paysans qui l'expérimentent et la font évoluer pour l'adapter à leur contexte agro-écologique et économique. Un suivi scientifique rigoureux de ces adaptations et des ateliers réflexifs avec les paysans permettent alors (iii) d'améliorer de manière itérative les techniques mises en œuvre. Cette approche produit des cycles d'apprentissage entre paysans, techniciens et chercheurs (Douthwaite et al., 2003) à partir d'une entrée technique initiale et conduit à accompagner les autres dimensions institutionnelles et organisationnelles tout au long du processus d'innovation.

En 2016, la découverte d'un procédé de bouturage assurant un taux de reprise satisfaisant débloque le premier obstacle 


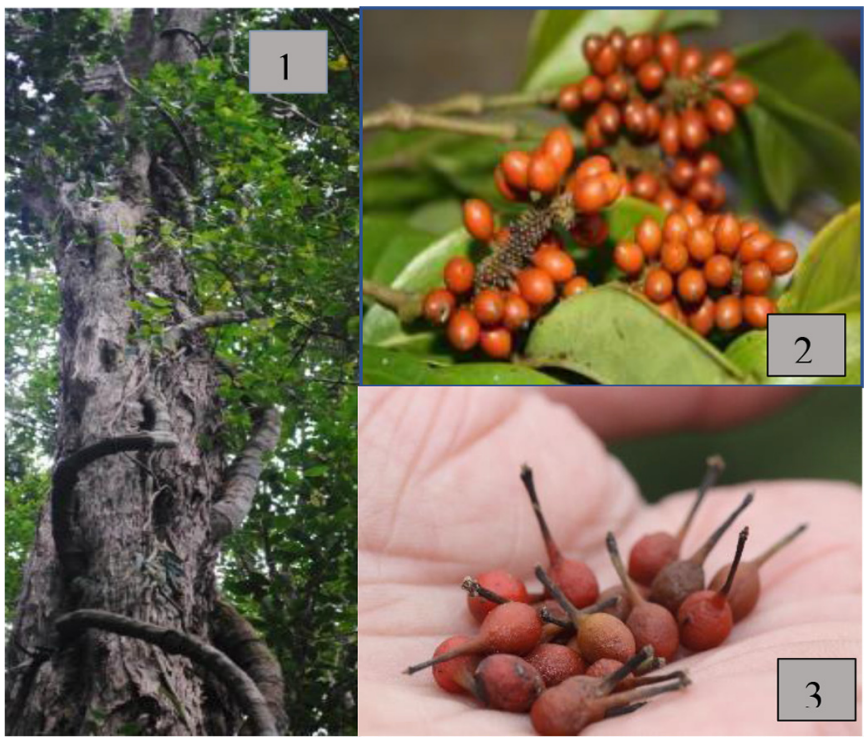

Fig. 1. Tsiperifery. Photo 1 : une liane sur son tuteur. Photo 2 : les baies fraîches. Photo 3 : les grains de poivre secs. (C) Razafimadimby. Fig. 1. Tsiperifery. Photo 1: a liana on its support tree. Photo 2: fresh berries. Photo 3: dried peppercorns.

technique à la culture du Tsiperifery: il devient alors possible de définir une première proposition d'itinéraire technique présentant des chances de succès que les chercheurs estiment raisonnables. Cet itinéraire est conçu par les chercheurs sur la base des résultats de recherche en cours, de l'expérience de planteurs privés et d'une étude bibliographique historique des procédés de domestication d'autres espèces de poivre (Ceccarelli et al., 2021).

Cet itinéraire technique constitue la «promesse plausible» initiale du processus de recherche participative. Il consiste à bouturer des lianes mâles et femelles. Les boutures sont prélevées sur des rameaux rampants de Tsiperifery sauvages. Les boutures des rameaux rampants ont un taux de survie plus élevé et leur prélèvement n'impacte pas la survie de la plante mère. Ces boutures sont entretenues en pépinières pendant environ six mois avant d'être transplantées.

Deux options de replantation sont alors envisagées : (i) la replantation en forêt dans l'écosystème d'origine des lianes mais dans des zones où les droits d'accès ne sont pas explicites ou (ii) la plantation sur tuteurs vivants au sein de parcelles agricoles privées, dans un écosystème différent mais dans un contexte foncier sécurisé. Les chercheurs suggèrent de planter un pied mâle au milieu d'un carré de huit femelles. Cette proposition s'inspire des mécanismes de pollinisation d'autres espèces de poivre (De Figueiredo et Sazima, 2004) et des résultats d'expériences de mise en culture du Tsiperifery conduites par des entreprises privées à Madagascar et devra faire l'objet d'une validation scientifique formelle.

Pour accompagner l'expérimentation de l'itinéraire technique, le programme s'appuie sur des associations paysannes dans les sites pilotes. Ces associations ont été créées et accompagnées tout au long des deux projets. En théorie, tous les villageois des sites d'intervention du programme de domestication du Tsiperifery pouvaient devenir membres de ces associations, à condition de participer à l'expérimentation et à la co-construction des techniques de culture du Tsiperifery. Un dispositif de suivi-évaluation mutualisé est mis en place pour observer la mise en œuvre des itinéraires techniques de domestication du Tsiperifery sur chaque site.

\subsubsection{Sites d'études}

L'étude a été menée dans trois sites de domestication du Tsiperifery à Madagascar (Fig. 2) :

- le fokontany Ambodivoangy, commune Ifanadina, région Vatovavy Fitovinany;

- le fokontany Ambodirafia, commune Kelilalina, région Vatovavy Fitovinany;

- la commune Ambongamarina, commune Ambongamarina, région Analamanga.

Les trois sites présentent des caractéristiques socioéconomiques similaires. Pour les trois sites, les principales activités sont la riziculture et l'élevage. La culture de bananiers est également importante à Ambodivoangy et Ambodirafia.

Sur ces trois sites, la cueillette est effectuée au sein de forêts domaniales gérées par le service du cantonnement forestier. Pour Ambodivoangy et Ambodirafia, les premières expériences de bouturage du Tsiperifery ont été initiées par un projet appelé Fararano mis en ouvre par l'ONG CRS, l'organisation NCBA CLUSA et le Centre de recherche ValBio en 2016. En 2017-2018, le projet Dometsip a poursuivi et accentué les actions déjà mises en place sur ces sites. Pour le cas de la commune Ambongamarina, le projet Capetsip a été mis en œuvre en 2017-2018 dans l'ensemble de la commune.

Les activités de culture du Tsiperifery réalisées par les membres des associations bénéficiaires des projets de domestication sur chaque site sont présentées dans le tableau 1 .

\subsection{Démarche d'analyse}

La méthode de collecte de données s'inspire de la grounded theory ou «théorie ancrée». Cette approche inductive consiste à construire des connaissances à partir des données empiriques obtenues sur le terrain (Cassell et Gillian, 2004). Sur les trois sites, des entretiens semi-structurés sont menés auprès de 90 paysans comprenant des membres des associations bénéficiaires des projets de domestication et des non-membres. Les thèmes abordés au cours de l'entretien portent sur les facteurs influençant les choix et la propension des paysans à tester et à co-construire l'itinéraire technique de mise en culture du Tsiperifery proposé par les porteurs du programme de domestication.

Les entretiens sont réalisés jusqu'à saturation de l'information. Pour les membres des associations bénéficiaires du programme de domestication du Tsiperifery, les entretiens sont réalisés avec la méthode d'échantillonnage par boule de neige. Pour les non-membres des associations, les interviewés ont été rencontrés de manière aléatoire dans les trois sites d'étude. La répartition des personnes interrogées est présentée dans le tableau 2. 


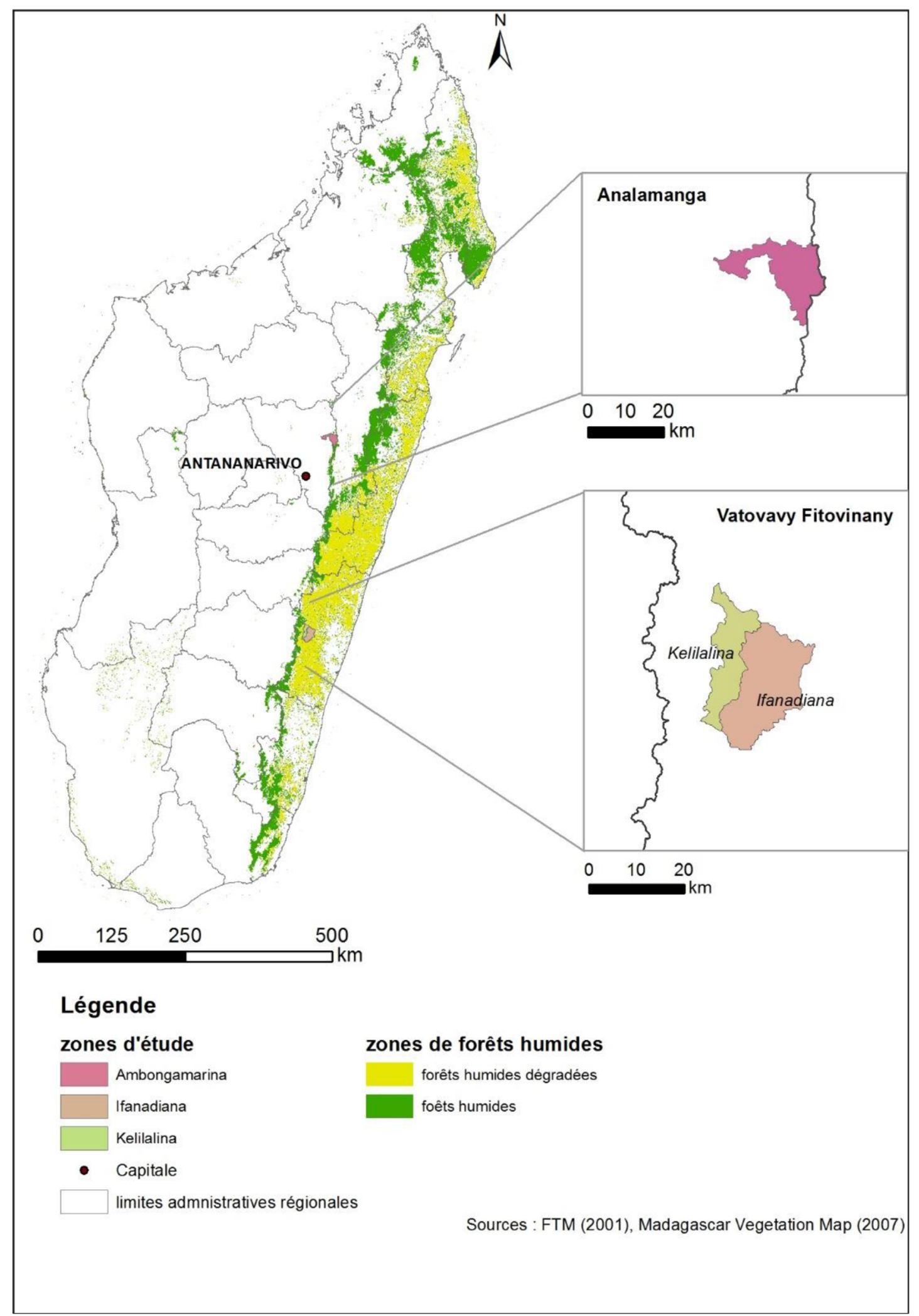

Fig. 2. Carte des sites d'étude.

Fig. 2. Map of the study sites. 
Tableau 1. Activités de bouturage et replantation du Tsiperifery réalisées sur les sites. Source: Document de suivi-évaluation des projets Dometsip et Capetsip.

Table 1. Cutting and replanting activities of Tsiperifery carried out at the sites.

\begin{tabular}{llllr}
\hline Sites & $\begin{array}{l}\text { Nombre de membres } \\
\text { des associations }\end{array}$ & $\begin{array}{l}\text { Nombre de boutures } \\
\text { sur pépinière }\end{array}$ & $\begin{array}{l}\text { Taux de survie des boutures } \\
(\%)\end{array}$ & $\begin{array}{l}\text { Nombres de boutures replantées } \\
(\%)\end{array}$ \\
\hline Ambodivoangy & 40 & 2836 & 60 & 1702 \\
Ambodirafia & 23 & 1845 & 42 & 775 \\
Ambongamarina & 241 & 14900 & 40 & 5960 \\
\hline
\end{tabular}

Tableau 2. Structuration de l'échantillon de paysans interrogés.

Table 2. Structure of the sample of surveyed farmers.

\begin{tabular}{llll}
\hline Sites d'études & Ambodivoangy & Ambodirafia & Ambongamarina \\
\hline $\begin{array}{l}\text { Nombre de membres des associations bénéficiaires } \\
\text { des projets de domestication du Tsiperifery }\end{array}$ & 12 & 8 & 26 \\
$\begin{array}{l}\text { Nombre de non-membres des associations } \\
\text { (non bénéficiaires des projets) }\end{array}$ & 13 & 7 & 24 \\
$\begin{array}{l}\text { Nombre de personnes interrogées } \\
\text { Total }\end{array}$ & 25 & 15 & 50 \\
\hline
\end{tabular}

Tableau 3. Exemple de codage ouvert.

Table 3. Example of open coding.

Texte retranscrit

... Avant l'arrivée du projet, nous en avons vendu à $500 \mathrm{Ar} \mathrm{le} \mathrm{kg} .$.

Depuis l'arrivée du projet Fararano et du responsable de la

société Jacarandas, nous étions certains qu'il y aurait un acheteur,

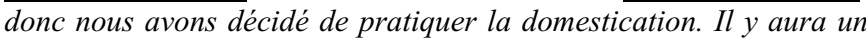

marché sûr pour l'écoulement du produit, et cela nous rapportera

un revenu considérable...

À partir de la retranscription des 90 entretiens, nous avons successivement procédé aux traitements suivants:

\section{- Étape 1 : Analyse textuelle}

Le texte de chaque entrevue est lu attentivement, les phrases clés sont soulignées. Les fragments de texte jugés pertinents sont ensuite codés en paraphrases soulignant les différentes idées transcrites (Tab. 3).

\section{- Étape 2 : Définition des concepts}

Les fragments codifiés énonçant les mêmes idées sont ensuite regroupés en concepts. Un exemple d'élaboration de concept est présenté dans le tableau 4.

\section{- Étape 3 : Classification en thèmes}

Les concepts sont ensuite regroupés en neuf thèmes qui seront présentés dans la partie suivante. Le choix du thème attribué à chaque groupe de concepts relève des connaissances et des expériences des chercheurs ayant effectué l'analyse des discours des interviewés.

\section{Codes}

- Arrivée du projet et Fararano et la société Jacarandas

- Assurance de l'existence d'acheteurs

- Source de revenu

Tableau 4. Exemple de regroupement en concepts.

Table 4. Example of grouping into concepts.

\begin{tabular}{ll}
\hline Codes & Concepts \\
\hline - Existence de marché & Marché sûr \\
- Venue d'un acheteur potentiel & \\
- Promesse d'achat par une société étrangère & \\
- Vente sans intermédiaire & \\
\hline
\end{tabular}

\section{- Étape 4 : Typologie comportementale des paysans}

Chaque personne interrogée a été référencée selon les thèmes principaux mentionnés dans son discours. Une typologie des comportements des paysans interrogés a ensuite été construite en regroupant les paysans présentant des profils de thèmes similaires. Cette typologie reflète les registres de justification de la logique déclarée vis-à-vis de l'adoption de la culture du Tsiperifery. 
Tableau 5. Facteurs de motivation pour adopter la technique.

Table 5. Motivational factors for adopting the technique.

\begin{tabular}{lllr}
\hline Thèmes & $\begin{array}{l}\text { Ambodivoangy } \\
(\%)\end{array}$ & $\begin{array}{l}\text { Ambodirafia } \\
(\%)\end{array}$ & $\begin{array}{l}\text { Ambongamarina } \\
(\%)\end{array}$ \\
\hline Logique économique & 100 & 88 & 92 \\
Communication et information & 50 & 75 & 19 \\
Confiance aux porteurs du projet & 50 & 25 & 0 \\
Accès au marché & 42 & 88 & 0 \\
Esprit de conservation & 42 & 25 & 69 \\
\hline
\end{tabular}

Tableau 6. Freins à l'adoption de la domestication du Tsiperifery.

Table 6. Barriers to adoption of Tsiperifery domestication.

\begin{tabular}{|c|c|c|c|}
\hline Thèmes & $\begin{array}{l}\text { Ambodivoangy } \\
(\%)\end{array}$ & $\begin{array}{l}\text { Ambodirafia } \\
(\%)\end{array}$ & $\begin{array}{l}\text { Ambongamarina } \\
(\%)\end{array}$ \\
\hline Faiblesse des réseaux de communication locaux & 0 & 14 & 8 \\
\hline Attente des retours des premières expériences & 77 & 57 & 71 \\
\hline Capacités productives et exclusion & 15 & 29 & 8 \\
\hline
\end{tabular}

\section{Résultats}

\subsection{Facteurs de succès et de blocage}

Suite à l'analyse de discours des paysans, 175 paraphrasescodes ont été identifiées. Elles ont permis d'élaborer 24 concepts dont un exemple est présenté dans le tableau 4. Le regroupement de ces concepts a conduit à neuf thèmes présentés dans les tableaux 5 et 6 . Ces tableaux indiquent également la proportion de paysans ayant fait référence à chacun des thèmes relatifs à la motivation ou aux freins à l'adoption de la mise en culture du Tsiperifery.

\subsubsection{Logique économique}

Avant l'arrivée du programme de domestication, la cueillette du Tsiperifery constituait une source de revenus saisonnière, mise à profit par quelques paysans pauvres en période de soudure. Ces derniers espèrent que la mise en culture de cueilli espèce permettra de pallier la raréfaction du Tsiperifery cueilli en forêt.

Pour les paysans qui ne pratiquent pas encore la cueillette en forêt, la mise en culture du Tsiperifery est également considérée comme une source d'amélioration de revenu. Le prix de vente élevé du produit, 20000 à $30000 \mathrm{MGA} / \mathrm{kg} \mathrm{sec}$ lors des enquêtes, constitue un facteur attractif important $(1 €=4480$ MGA $)$.

De plus, la possibilité d'association du Tsiperifery avec d'autres cultures permet aux paysans d'envisager une diversification de leurs activités sans renoncer à une culture déjà pratiquée.

\subsubsection{Communication et information}

La quantité et la qualité des informations sur la mise en culture du Tsiperifery, reçues de bouche à oreille au sein de la communauté ou via les sensibilisations des techniciens, constituent des facteurs de motivation.

La participation des paysans aux foires nationales de l'agriculture (en 2017 et 2018) a notamment eu des effets importants. Les paysans ayant participé à ces foires ont pu constater l'existence de nombreux acteurs intéressés par la filière au niveau national. De même, les visites des techniciens agricoles, des chercheurs et des différents agents du programme, ont permis aux paysans d'acquérir plus d'informations sur cette filière.

À l'inverse, l'absence d'information ou leur qualité jugée insuffisante constituent des freins. Ce déficit d'information est corrélé à une faible intégration dans les réseaux de communication locaux. Selon des non-membres des associations interrogés à Ambodirafia et Ambongamarina, les associations accompagnées par les projets sont exclusives et communiquent peu avec le reste de la communauté. Les personnes n'ayant pas de relation directe avec les présidents de ces associations n'ont pas été informées de la création de ces dernières et ne reçoivent pas d'information technique ou sur l'évolution des prix d'achat du Tsiperifery. Néanmoins, il est noté que seuls $14 \%$ des interviewés à Ambodirafia et $8 \%$ à Ambongamarina ont évoqué ce problème.

\subsubsection{Maintien de bonnes relations avec les acteurs du développement}

À Ambodivoangy et Ambodirafia, plusieurs paysans disent avoir expérimenté la culture du Tsiperifery du fait de la proximité entre ce programme de domestication, le centre de recherche ValBio et le projet Fararano, deux institutions locales ayant développé depuis de nombreuses années des relations de confiance avec ces villages. La plupart des paysans ayant accepté l'expérimentation de la culture du Tsiperifery sur ces sites ont déjà bénéficié de plusieurs actions de 
Tableau 7. Typologie des adoptants potentiels selon leur logique d'adoption de la domestication du Tsiperifery. Table 7. Typology of potential adopters according to their logic of adoption of Tsiperifery domestication.

\begin{tabular}{|c|c|c|c|}
\hline Sites/Types de comportement & $\begin{array}{l}\text { Ambodivoangy } \\
(\%)\end{array}$ & $\begin{array}{l}\text { Ambodirafia } \\
(\%)\end{array}$ & $\begin{array}{l}\text { Ambongamarina } \\
(\%)\end{array}$ \\
\hline «Homo-economicus» & 8 & 7 & 14 \\
\hline «Conservationnistes» & 20 & 13 & 36 \\
\hline «Exclus $»$ & 8 & 20 & 8 \\
\hline
\end{tabular}

développement portées par ces acteurs. Ils ont accepté d'expérimenter cette technique notamment pour entretenir leur relation avec ces acteurs du développement.

\subsubsection{Accès au marché}

L'existence d'un circuit commercial fiable et établi pour l'écoulement du Tsiperifery constitue une motivation importante. La venue d'un représentant d'une société exportatrice d'épices à Ambodirafia et Ambodivoangy et une promesse d'achat ont eu un effet incitatif important.

\subsubsection{Esprit de conservation}

Certains paysans considèrent la culture du Tsiperifery comme un instrument de conservation de la forêt. La replantation de plants en forêts est en effet susceptible de transformer les règles d'accès aux ressources forestières. Les zones de replantation de Tsiperifery seront protégées par les propriétaires des lianes, ce qui bénéficiera à la forêt. D'autres paysans justifient leur adhésion au programme en se référant aux générations futures à qui ils espèrent laisser des ressources forestières en bon état et des pieds de Tsiperifery productifs.

\subsubsection{Aversion au risque}

L'aversion au risque regroupe les notions de risque et d'incertitude. Les paysans interrogés doutent de l'existence d'un marché pour ce produit et craignent que les lianes ne fructifient pas, que la fructification prenne trop de temps ou que les prix s'effondrent une fois que la domestication aura permis d'augmenter la production. Ces fortes incertitudes les conduisent à adopter une stratégie d'attente afin de limiter les risques; ils n'adoptent donc pas ces itinéraires techniques.

Certains paysans disent ne pas avoir de temps à consacrer à cette pratique mais disent vouloir participer plus tard à la culture du Tsiperifery. Ils souhaitent avoir les retours d'expérience des premiers paysans participant à la domestication du Tsiperifery. Ils attendent que le processus expérimental soit achevé et les itinéraires techniques stabilisés.

\subsubsection{Capacités productives et exclusion d'acteurs}

À Ambodirafia, le problème de l'accès au foncier reste un facteur de blocage pour les paysans arrivés récemment. Sur ce site, le choix a été fait d'une plantation uniquement sur des parcelles agricoles à usage commercial. Ce choix exclut du projet les migrants, qui n'ont pas un accès foncier sécurisé sur le long terme par le droit coutumier et n'ont pas d'arbres tuteurs sur leurs parcelles. Ce n'est pas le cas pour les sites Ambongamarina et Ambodivoangy, où les membres des associations replantent les boutures sur des parcelles communes et/ou sur des parcelles privées, ce qui permet la participation des migrants au projet.

Le manque de temps et de ressources financières fait aussi partie des freins à l'adoption. Pour obtenir des formations et du matériel, les paysans doivent adhérer à une association bénéficiaire du programme, donc participer à des réunions et à des travaux collectifs. Or, les salariés agricoles ont peu de temps à consacrer à ces activités, qui représentent autant de journées rémunérées de perdues. Cela a été remarqué pour des paysans du site Ambodivoangy. Le règlement interne de l'association accentue également ce problème, car les absents aux réunions ou aux travaux collectifs doivent payer 2000 MGA par absence, ce qui est une somme importante pour un salarié agricole qui ne gagne que 3000 à $3500 \mathrm{MGA}$ par jour.

\subsection{Typologie des adoptants potentiels}

En se référant aux facteurs influençant les décisions des paysans à participer ou non au processus de domestication du Tsiperifery, les participants potentiels à ce programme peuvent être catégorisés en cinq types: les «homo-economicus», les « conservationnistes», les « opportunistes», les « exclus du processus » et les «suiveurs ». Les proportions de chaque type au niveau de chaque zone d'étude sont présentées dans le tableau 7.

Les arguments relatifs à la logique économique se retrouvent parmi tous les types d'adoptants. Nous avons qualifié d' «homo-economicus» ceux qui mobilisent uniquement ce registre de justification. Ils sont minoritaires dans les trois sites d'études (14\% pour le maximum, à Ambongamarina). Les raisons de la participation de ce groupe au processus de domestication répondent aux modèles ricardiens de comportement optimal visant à maximiser une utilité (Pareto, 1909). Les adoptants d'un deuxième type sont qualifiés d' "opportunistes ». Ce sont des paysans habitués à profiter des retombées des projets de développement. Il est plausible que leur attrait pour 
la culture de Tsiperifery évoluera une fois les projets achevés. Ils sont surtout présents à Ambodivoangy et Ambodirafia. Il existe également des paysans «conservationnistes », qui justifient leur participation au programme de domestication par souci de conservation de leurs ressources forestières. Ils sont plus nombreux à Ambongamarina. Il existe aussi des paysans qui peuvent être considérés comme étant «exclus » du processus de recherche participative. Ce sont les paysans qui disent ne pas avoir été informés lors de la création des associations, ainsi que ceux qui ne peuvent pas participer à l'expérimentation de la culture du Tsiperifery faute de moyens productifs : accès sécurisé au foncier pour les migrants ou temps libre pour les ouvriers agricoles. Les « suiveurs » sont les paysans qui attendent de voir les résultats des premiers expérimentateurs avant de prendre une décision; ils agissent principalement par imitation: ils n'adopteront les innovations que lorsque ces dernières auront été testées et seront devenues courantes au sein de la communauté.

\section{Discussion}

\subsection{Amorce de compréhension du processus de domestication du Tsiperifery}

La logique économique est présente dans les discours de la majorité des paysans dans chacun des trois sites. Toutefois, ce n'est pas le principal facteur influençant les décisions des participants au programme de domestication. Les principaux registres de justification évoqués par les paysans diffèrent selon les sites: la communication et la confiance dans les porteurs du projet à Ambodivoangy, la communication et l'accès au marché à Ambodirafia, et la conservation des ressources forestières à Ambongamarina. Ces différences soulignent l'importance des facteurs socio-économiques et écologiques: les forces des interactions et des relations anciennes entre les porteurs du programme de domestication et les paysans pour Ambodivoangy; l'accès au marché via la venue d'un acheteur promettant l'achat du Tsiperifery pour Ambodirafia; la prise de conscience des paysans de la raréfaction des ressources naturelles pour Ambongamarina. Dans ce dernier cas, la forte dégradation des forêts a conduit les paysans à conserver les fragments de forêts restant dans la zone; un phénomène similaire a été observé à Madagascar par Hervé et al. (2020).

En ce qui concerne la typologie construite, à l'inverse de celles couramment utilisées dans les études de diffusion des innovations inspirées des catégories de Rogers (1983) (pionniers, innovateurs, majorité précoce, majorité tardive et retardataires), notre typologie ne reflète pas de changement de profil d'adoptant en fonction de la dynamique d'adoption. Cela peut s'expliquer par le fait que le processus de domestication du Tsiperifery est très récent et que tous les types que nous avons identifiés correspondent aux phases initiales d'émergence de l'innovation. Il est probable que cette typologie tendra à se complexifier au fur et à mesure de l'avancée de la dynamique d'innovation. Les paysans qui ont accepté les premiers de participer au processus de domestication pourraient constituer les pionniers de cette innovation.

À l'instar des typologies d'adoptants de l'innovation comme celle de Audouin et Gazull (2014), la typologie de cette étude traduit une diversité de représentations à l'échelle individuelle. Or, les déterminants de l'adoption de la culture du Tsiperifery combinent des déterminants qui se réfèrent à une échelle individuelle (comme l'amélioration des revenus des ménages, les capacités productives de l'exploitation ou l'accès aux projets de développement), mais aussi des déterminants qui se réfèrent à une échelle collective (le réseau de communication, l'accès au marché, l'état de dégradation des ressources forestières, ou les jeux de pouvoir autour du contrôle des associations). Ce résultat souligne l'importance de la prise en compte de l'ensemble des éléments qui composent un système d'innovation dans les dispositifs d'accompagnement d'une innovation (Toillier et al., 2018) et dans un territoire donné (Audouin et Gazull, 2014).

Néanmoins, la typologie construite offre une compréhension empirique approfondie des représentations, des motivations et des freins à la participation des paysans au programme de domestication du Tsiperifery. Ce sont des éléments à prendre en compte dans la conduite future du programme de domestication, notamment l'exclusion de certains types de paysans (les migrants et les ouvriers agricoles), l'importance de la perception de l'état de dégradation de la forêt, et l'importance d'apporter des preuves sur les performances techniques de la production du Tsiperifery. Selon les sites, les actions d'accompagnement pourront être adaptées dans une perspective de gestion territoriale agricole et forestière.

\subsection{Incertitudes techniques sur la domestication du Tsiperifery}

Le manque de données et d'études concernant la phénologie et l'itinéraire technique de domestication du Tsiperifery invite à la prudence et à s'interroger sur la pertinence de la diffusion de cette pratique à ce stade des connaissances scientifiques. Les domaines d'exploration scientifique sont nombreux : mode de propagation, fructification, rendement, maintien de la diversité génétique, qualités organoleptiques du Tsiperifery domestiqué, ravageurs et parasites de la plante. Dans le cas de la domestication de l'hévéa en Amazonie, les espèces plantées sur parcelle agricole ont été atteintes par des maladies de brûlure des feuilles (Boot, 1997). Un autre exemple est le cas de la domestication de Aquarilla spp. produisant du bois d'agar au Laos, un PFNL très intéressant au niveau économique (Jensen, 2004). Les arbres domestiqués ont produit un bois d'agar de moins bonne qualité que ceux de la forêt naturelle. Ces innovations, conçues pour résoudre un problème donné, ont dû affronter, in itinere, de nouveaux problèmes (Baret, 2014). Ainsi, des problèmes pourraient survenir pour les plantations sur parcelles agricoles du Tsiperifery, vu le manque d'informations précises sur les pollinisateurs ou les risques de maladies. Il reste donc indispensable de s'assurer que les observations techniques effectuées sur les sites pilotes de domestication soient discutées au sein d'un dispositif multi-acteurs d'accompagnement de l'innovation afin d'adapter les techniques in itinere et d'accompagner la résolution des problèmes techniques, institutionnels et organisationnels qui ne manqueront probablement pas de survenir. 


\section{Conclusion}

Cette étude met en évidence les logiques comportementales des paysans dans le choix de participer au processus de co-construction de connaissances dans le cadre d'un programme de domestication du Tsiperifery. La recherche d'avantages possibles comme le profit se retrouvent dans les raisons d'adoption de la culture du Tsiperifery exprimées par les paysans interrogés. Néanmoins, pour expliquer leurs décisions, la plupart évoquent aussi, en plus de cet argument économique, d'autres raisons, telles que le souci de la conservation des ressources forestières ou le maintien des relations avec les porteurs du projet. Les participants centrés uniquement sur une logique économique sont minoritaires dans les trois sites d'études. Le manque d'informations sur la production et le marché reste un facteur de blocage à l'adoption de la culture du Tsiperifery. Il a été également remarqué que la mise en place du programme de domestication exclut des groupes sociaux comme les migrants et les ouvriers agricoles.

Cette étude apporte des éléments de réflexion aux acteurs du développement et de la conservation pour mettre en œuvre des mesures d'accompagnement tenant compte de la diversité des logiques comportementales des paysans des sites d'études. Elle appelle également à ne pas se limiter à une analyse au niveau individuel. Une analyse au niveau du système d'innovation permet de rendre compte des dimensions techniques, économiques et institutionnelles du système, mais aussi des caractéristiques des territoires, où l'état de dégradation des forêts est perçu différemment par les paysans. Le manque d'informations techniques sur la mise en culture du Tsiperifery peut également constituer un facteur non négligeable freinant les paysans pour tester et mettre en pratique ces solutions techniques.

\section{Références}

Arnold JE, Ruiz Pérez M. 2001. Can non-timber forest products match tropical forest conservation and development objectives? Ecological Economics 39(3): 437-447. DOI: 10.1016/S0921-8009 (01)00236-1.

Audouin S, Gazull L. 2014. Les dynamiques d'un système d'innovation par le prisme des diffusions spatiales: le cas de l'anacarde au Sud-Ouest du Burkina Faso. L'Espace Géographique 43(1): 35-50. DOI: 10.3917/eg.431.0035.

Baret P. 2014. Agroecology and Innovation: Semantic games and variety of designs for which transitions? Fourrages 217: 101-103.

Belcher B, Ruiz-Perez M, Achdiawan R. 2005. Global patterns and trends in the use and management of commercial NTFPs: Implications for livelihoods and conservation. World Development 33(9): 1435-1452. DOI: 10.1016/j.worlddev.2004.10.007.

Bertrand A, Aubert S, Montagne P, Lohanivo AC, Razafintsalama MH. 2014. Madagascar, Politique Forestière : Bilan 1990-2013 et Propositions. Madagascar Conservation \& Development 9(1): 2030. DOI: $10.4314 / \mathrm{mcd} . v 9 i 1.4$.

Boot RG. 1997. Extraction of non-timber forest products from tropical rain forests. Does diversity come at a price? Netherlands Journal of Agricultural Science 45(4): 439-50. DOI: 10.18174/ njas.v45i4.504.
Cassell C, Gillian S. 2004. Essential guide to qualitative methods in organizational research. London (GB): SAGE Publications, 409 p. DOI: $10.4135 / 9781446280119$.

Ceccarelli V, Queste J, Barbieri J. 2021. Towards domestication of the endemic Malagasy pepper Tsiperifery (Piper sp.): Lessons learnt from domestication and cultivation of other peppers. Fruits, accepted.

Danthu P, Razakamanarivo RH, Deville-Danthu B, Razafy Fara L, Le Roux Y, Penot E. 2016. The short and forgotten history of rubber in Madagascar: The first controversy between biodiversity conservation and natural resource exploitation. Bois et Forêts des Tropiques 328: 27-43. DOI: 10.19182/bft2016.328.a31300.

De Figueiredo RA, Sazima M. 2004. Pollination ecology and resource partitioning in Neotropical Pipers. In: Dyer LA, Aparna D, Palmer N, eds. Piper: A Model Genus for Studies of Phytochemistry, Ecology, and Evolution. New York (USA): Kluwer Academic, pp. 33-57. DOI: 10.1007/978-0-387-30599-8 3.

Douthwaite B, Kuby T, Van De Fliert E, Schulz S. 2003. Impact pathway evaluation: An approach for achieving and attributing impact in complex systems. Agricultural Systems 78(2): 243-265. DOI: 10.1016/S0308-521X(03)00128-8.

Hervé D, Randriambanona H, Ravonjimalala HR, Ramanankierana H, Rasoanaivo NS, Baohanta R, et al. 2020. Perceptions des fragments forestiers par les habitants des forêts tropicales humides malgaches. Bois et Forêts des Tropiques 345: 43-62. DOI: 10.19182/bft2020.345.a31929.

Jensen A. 2004. Domestication of Aquilaria spp. and rural povertysocio-economic and genetic aspects of the planting boom in "Wood of the Gods". In: Poverty Reduction and Shifting Cultivation Stabilization in the Uplands of Lao PDR: Technologies, Approaches and Methods for Improving Upland Livelihoods. Proceedings of a Workshop held in Luang Prabang, Lao PDR, pp. 233-239.

Kusters K, Achdiawan R, Belcher B, Ruiz-Pérez M. 2006. Balancing development and conservation? An assessment of livelihood and environmental outcomes of non-timber forest product trade in Asia, Africa, and Latin America. Ecology and Society 11(2): 20. DOI: 10.5751/ES-01796-110220.

Palchetti E, Biricolti S, Gori M, Rota Nodari G, Gandolfi N, Papini A. 2018. Two new Malagasy species of genus Piper L. (Piperaceae), Piper malgassicum and Piper tsarasotrae, and their phylogenetic position. Turkish Journal of Botany 42: 610-622. DOI: 10.3906/ bot-1712-2.

Pareto V. 1909. In: Giard V, Brière E, eds. Manuel d'économie politique [Traduit par Bonnet A. 5th ed.]. Paris (France): Bibliothèque internationale d'économie politique, $695 \mathrm{p}$.

Razafimandimby H, Benard AG, Andrianoelisoa H, Leong Pock Tsy JM, Touati G, Levesque A, et al. 2017. Tsiperifery, the wild pepper from Madagascar, emerging on the international spice market whose exploitation is unchecked: Current knowledge and future prospects. Fruits 72(6): 331-340. DOI: 10.17660/th2017/72.6.1.

Rogers EM. 1983. Diffusion of innovations, 3th ed. USA: Macmillian Publishing Co., 453 p.

Stewart KM. 2003. The African cherry (Prunus africana): Can lessons be learned from an over-exploited medicinal tree? Journal of Ethnopharmacology 89(1): 3-13. DOI: 10.1016/j. jep.2003.08.002.

Tchatat M, Ndoye O. 2006. Étude des produits forestiers non ligneux d'Afrique centrale: réalités et perspectives. Bois et Forêts des Tropiques 288(2): 27-39.

Toillier A, Devaux-Spatarakis A, Faure G, Barret D, Marquié C. 2018. Comprendre la contribution de la recherche à l'innovation collective par l'exploration de mécanismes de renforcement de capacité. Cahiers Agricultures 27(1): 13. DOI: 10.1051/cagri/2017055. 
Touati G. 2012. État des lieux de la gestion du poivre sauvage de Madagascar. Un produit forestier non ligneux exploité pour la commercialisation. Mémoire de fin d'étude, ISTOM, France, $78 \mathrm{p}$.
Vantomme P, Gazza S. 2010. Le défi de la sylviculture en faveur des produits forestiers non ligneux sous les tropiques : de la cueillette à l'agriculture? Bois et Forêts des Tropiques 304(2): 5-13. DOI: 10.19182/bft2010.304.a20447.

Citation de l'article : Rasambo SN, Queste J, Razafiarijaona J, Audouin S, Jankowski F, Rabefarihy T, Ramananarivo R. 2021. Stratégies paysannes de participation à la domestication du poivre sauvage de Madagascar, le Tsiperifery. Cah. Agric. $30: 24$. 